\title{
Percepción del adulto mayor sobre la calidad de vida en los hoga- res de ancianos de Tegucigalpa, 2014
}

\author{
Darguin Manuel Amaya Varela ${ }^{1}$ \\ Elvis Geovany Trejo Teruel ${ }^{2}$
}

\section{RESUMEN}

En el presente artículo se aborda la temática de los adultos mayores viviendo en los hogares de ancianos, teniendo como objetivo principal conocer cuál es la percepción que tienen los adultos mayores acerca de la calidad de vida en los hogares de ancianos de Tegucigalpa en el año 2014.

El instrumento utilizado fue una entrevista estructurada y la observación participante, la muestra fue por conveniencia y estuvo compuesta de 22 adultos; 10 mujeres y 12 hombres, todos mayores de 65 años y que viven dentro de los hogares de ancianos Salvador Aguirre, María Eugenia, Hilos de Plata y Centro de Día y Reposo (CEDER).

La investigación se enmarca dentro de un enfoque cualitativo, partiendo de la perspectiva de los participantes en un ambiente natural y con relación al contexto, basada en un diseño fenomenológico, el cual se enfoca en las experiencias individuales subjetivas de los participantes y desde la perspectiva construida colectivamente; para facilitar el análisis y la interpretación de datos cualitativos se utilizó el programa de atlas ti.

Como resultado se puede decir que los adultos mayores consideran que tienen buena calidad de vida en comparación con la que tenían antes de llegar al hogar de ancianos y son pocos los que son visitados por sus familias.

Palabras clave: adulto mayor, familia, calidad de vida, hogar de ancianos.

\footnotetext{
${ }^{1}$ Beneficiario de beca de estudiante de posgrado de la DICYP, Maestría en Metodologías de Investigación Económica y Social, UNAH: darwinamaya94@yahoo.es

${ }^{2}$ Asesor, Profesor, Escuela de Sociología, Facultad de Ciencias Sociales, UNAH: historiahn@yahoo.com
} 


\section{ABSTRACT}

The article's main objective is to find out what elderly think in regard to the quality of life in nursing homes in Tegucigalpa in 2014, therefore a group of seniors who live in nursing homes were interviewed.

The instruments used were structured interviews and participant observations. The sample was chosen by convenience, being composed of 22 people in total, 10 women and 12 men, all over 65 years old and living in the following nursing homes: Salvador Aguirre, Maria Eugenia, Silver Threads and Day Centre and Rest (ASSIGN).

The research is part of a qualitative approach, based on the perspective of the participants in a natural environment and in relation to the context, based on a phenomenological design, which focuses on the subjective individual experiences of the participants and a collective perspective. The program Atlas Ti was used to facilitate the analysis and interpretation of qualitative data.

As a result we can say that older adults feel that they have a good quality of life in comparison to their situation before reaching the nursing home. Nonetheless, only a few of them are visited by their families.

Keywords: elderly, family, quality of life, nursing home. 


\section{INTRODUCCIÓN}

El propósito general de la investigación fue conocer la percepción que tienen los adultos mayores acerca de la calidad de vida en los hogares de ancianos: Salvador Aguirre, María Eugenia, Hilos de Plata y Centro de Día y Reposo (CEDER) de Tegucigalpa. Esta investigación con adultos mayores en los hogares de ancianos surge a partir de que actualmente en nuestro país la población mayor de sesenta años ha tenido un gran crecimiento sobre el total de la población, con esta investigación se pretende exponer la gran cantidad de adultos mayores que se enfrentan a una problemática cada vez más creciente dentro de la sociedad y esta se trata del olvido en el que está viviendo el adulto mayor.

En esta investigación se logró identificar cómo se sienten los adultos mayores en los hogares de ancianos, ya que muchas veces al ingresar a estas residencias, los adultos presentan diferentes situaciones que afectan su calidad de vida, como la separación de la familia, lo que genera en algunos sensaciones de abandono, exclusión, depresión, estrés y algunas veces enfermedades físicas y mentales, como consecuencia de la separación de su familia. Este es un cambio que muchas veces afecta, porque deben integrarse a un nuevo grupo social, el cual es manejado y administrado con diferentes normas y maneras de convivencia.

La información proporcionada por los adultos mayores se ve reflejada de manera textual y a través de la observación se encontraron algunas dificultades en el proceso de investigación, estas se fueron desarrollando con precisión y algunos de los aspectos que se superaron fueron: poca apertura para la investigación por parte de los administradores de los hogares de ancianos, también hay varios adultos mayores que no están en la capacidad de brindar entrevistas debido a algunos problema de salud o mental; además, en los hogares de ancianos brindan información desactualizada, todo esto se pudo superar a medida que se avanzó con la investigación.

\section{OBJETIVOS}

\section{Objetivo general}

Conocer cuál es la percepción que tienen los adultos mayores acerca de la calidad de vida en los hogares de ancianos: Salvador Aguirre, María Eugenia, Hilos de Plata y 
Centro de Día y Reposo (CEDER) de Tegucigalpa, de junio a septiembre de 2014.

\section{Objetivos específicos}

1. Identificar las prácticas que llevan a cabo las familias y los hogares de ancianos para brindarle calidad de vida al adulto mayor en hogares de ancianos: Salvador Aguirre, María Eugenia, Hilos de Plata y Centro de Día y Reposo (CEDER) de Tegucigalpa, junio-septiembre de 2014.

2. Describir el perfil del recurso humano que atiende a los adultos mayores en los hogares de ancianos: Salvador Aguirre, María Eugenia, Hilos de Plata y Centro de Día y Reposo (CEDER) de Tegucigalpa, junio-septiembre de 2014.

3. Identificar las principales necesidades físicas, sociales, económicas y emocionales del adulto mayor en los hogares de ancianos: Salvador Aguirre, María Eugenia, Hilos de Plata y Centro de Día y Reposo (CEDER) de Tegucigalpa, junio-septiembre de 2014.

4. Determinar algunas causas por las cuales los adultos mayores están en los hogares de ancianos: Salvador Aguirre, María Eugenia, Hilos de Plata y Centro de Día y Reposo (CEDER) de Tegucigalpa, junio-septiembre de 2014.

\section{METODOLOGÍA}

El enfoque de esta investigación es cualitativo, en la cual se pretende hacer una aproximación sistémica que permita describir las experiencias de la vida y darles significado desde la perspectiva de los adultos mayores. La investigación cualitativa se enfoca en comprender y profundizar los fenómenos, explorándolos desde la perspectiva de los participantes en un ambiente natural y con relación al contexto.

Con base al problema planteado sobre analizar algunos factores que inciden en la calidad de vida de los adultos mayores residentes en los hogares de ancianos en Tegucigalpa y en función de los objetivos planteados, la presente es una investigación descriptiva, caracterizada por destacar "...Las características de un tipo de gestión, conducta de un individuo o conductas grupales, de la comunidad, así como la descripción de las características de una determinada comunidad o situación" (Balestrini, 2000, p.6). 
En cuanto al contexto, se realizó bajo una investigación observacional, ya que se abordaron los hechos bajo condiciones naturales, en vista de que los hechos ya existen en la realidad, lo que hace el investigador es estar de acuerdo y reconocer. Para esto, Yuni, J.A. y Urbano, C.A. (2003) plantean la necesidad de tomar una muestra, observando una fracción de unidades, obteniendo resultados parecidos a los que lograríamos si estudiaríamos el universo total, en pocas palabras es encontrar una forma de obtener información relevante sin la necesidad de acudir a la medición de todo el universo posible.

La investigación se realizó basada en un diseño fenomenológico, este diseño se enfoca en las experiencias individuales subjetivas de los participantes. Responden a la pregunta sobre cuál es el significado, estructura y esencia de una experiencia vivida por una persona individual, grupo o comunidad, con respecto a un fenómeno. El centro de indagación de estos diseños reside en las experiencias de los participantes.

La fenomenología es originalmente una filosofía encargada de esclarecer el paradigma de cómo conocernos e interesada en los atributos de la experiencia consciente. Castro (2011, p. 93) sostiene que Edmund Husserl es uno de los precursores de la fenomenología y su teoría agrega que:

La vía de acceso a las respuestas para estas preguntas es la meditación reflexiva, liberada de toda prenoción o compromiso con cualquier marco teórico o sistema de categorías preexistente, con el fin de acceder a la esencia de la conciencia, a sus constantes universales, más allá de sus manifestaciones históricas concretas.

En la fenomenología, los fenómenos son las cosas naturales tal como se muestran, como se ofrecen a la conciencia; se interesa por la conciencia, entendida esta como el estado en el que se hace presente la realidad, a la que llama fenómeno. Esta teoría fue desarrollada por el filósofo alemán Edmund Husserl (1859-1938), quien postula que el investigador debe describir la experiencia como se da, desprovista de apriorismos o interpretaciones.. Así, pues, la cualidad del buen fenomenólogo es la perfección en el mirar, es decir, abordar los fenómenos con una disposición desprejuiciada, lo que hará posible que capte de las realidades lo que tienen de propias, sin añadidos personales. (Báez, Juan y De Tudela, Pérez, 2009)

Las técnicas con las que trabajan son: la observación pura para tener acceso a las realidades y poderlas conocer. La observación debe hacerse desde la contemplación y con diálogo e interrogación con los actores del tema investigado, como escucha 
activa, reflexiva, como análisis. La otra técnica es la descripción rigurosa, que es el modo en el que da a conocer las realidades; el investigador ha de desterrar en su narración cualquier juicio de valor que pudiera haberse formado sobre el fenómeno sometido a estudio.

Para esta investigación la población o las unidades de análisis de observación 0 estudio fueron la totalidad de los adultos mayores residentes en los 13 hogares de ancianos del Distrito Central, en los que viven 251 adultos mayores, todos ellos constituyeron la población universo de estudio para la investigación planteada, para los cuales se generalizaron los resultados, entendiendo que el universo o población está formado por todo el conjunto de unidades estudiadas y que podrían ser observadas individualmente en el estudio.

Por definición, una muestra es una parte del universo de las unidades de análisis del estudio que permite obtener información sobre esa totalidad. La muestra cualitativa tiene la forma de un espiral que se contrae y vuelve sobre si, como círculo virtuoso de información (Escribano, 2007).

Teniendo como población la totalidad de los adultos mayores que residen en los hogares de ancianos en el Distrito Central, se diseñó la muestra por conveniencia para escoger cuatro hogares de ancianos: Salvador Aguirre (7 entrevistas), María Eugenia (7 entrevistas), Hilos de Plata (7 entrevistas) y Centro de Día y Reposo (1 entrevista), en cada uno de ellos se realizaron las entrevistas a ambos sexos, a cada director de las instituciones y dos entrevistas al personal que trabaja para los hogares de ancianos y que pasa mucho tiempo con los adultos mayores.

En esta investigación se utilizó el muestreo de conveniencia, que es un diseño en el que se seleccionan aquellos sujetos más fácilmente accesibles. La muestra por conveniencia es el procedimiento que consiste en la selección de las unidades de la muestra en forma arbitraria; estas se autoseleccionan o se eligen de acuerdo a su fácil disponibilidad (Mejía Navarrete, 2002).

En cuanto a las técnicas que se utilizaron para poder cumplir con los objetivos propuestos, fue necesaria la aplicación de una entrevista estructurada, cuyo propósito fue conocer aspectos personales básicos de cada uno de los entrevistados; también se tomó en cuenta para la obtención de la información: la observación, investigación bibliográfica y registro fotográfico. Para la realización de este proyecto se utilizaron las siguientes técnicas: entrevista semiestructurada y observación participante. 
Para la recolección de datos se aplicaron entrevistas grabadas y trascritas para el análisis de los resultados, también se realizó un sistema de revisión de la información obtenida en cada una de las entrevistas, resaltando los aspectos importantes e identificándolas de acuerdo con las variables y objetivos elaborados previamente.

El trabajo se abordó a través de la recopilación de datos por medio de encuestas y entrevistas que se aplicaron a tres grupos para comparar los resultados de las opiniones brindadas por los adultos mayores, como primer grupo; el segundo grupo lo conforman el personal de apoyo como enfermeras, médicos, aseadoras, conserjes, personal de cocina y todas las personas que tengan relación con los adultos mayores; el tercer grupo son los directores, administradores, asistentes, gerentes o dueños de los hogares de ancianos.

Como es un análisis cualitativo, con esta metodología se permite conservar el lenguaje original de los sujetos, indagar su definición de la situación, la visión que tiene de su propia historia y de sus condiciones en los hogares. Además para este análisis se utilizaran fragmentos de lo expresado en las entrevistas por los adultos mayores, identificados con un código con la inicial del hogar de ancianos, sexo, número y fecha de entrevista.

Con el fin de obtener resultados determinantes de los datos recabados, se usó el software Atlas ti en el proceso de análisis y codificación en esta investigación, mediante el cual se obtienen tablas y gráficas, simplificando el contenido de los datos y dando como resultado la información necesaria para el estudio.

\section{ANÁLISIS DE DATOS}

De acuerdo con los resultados obtenidos en la presente investigación, se han observado varios aspectos importantes que se detallarán a continuación. Entre los aspectos generales se puede identificar que la mayoría de los adultos mayores en los hogares de ancianos en Tegucigalpa tiene arriba de los 70 años y que el mayor número de residentes son mujeres, de acuerdo con los listados de cada hogar y las entrevistas realizadas.

En su mayoría los adultos mayores están viudos y otros que nunca se casaron. En cuanto al tiempo de permanecer en los hogares de ancianos, se puede decir que es variado, ya que el periodo de tiempo está entre los 6 meses y 20 años (Hace 7 años 
me trajeron del Chiverito, ahí pasaba porque no tenía donde vivir y me gustaba beber. Entrevista a Darguin Amaya, código de entrevistado: CEAM1, Tegucigalpa M.D.C, 19 de diciembre 2014.

La mayoría de los adultos mayores entrevistados saben leer y escribir, lo que se significa que la escolaridad no es una condición suficiente para tener el sustento diario en casa, ni tampoco para la plena integración de las personas en la sociedad; sin embargo, si contribuye a tener un mejor estilo de vida dentro de los hogares de ancianos, ya que favorece su crecimiento cultural, amplía su posibilidad de esparcimiento mediante la lectura y favorece su independencia (Sí, yo soy bachiller, me gradué del Instituto Normal Central de Varones, ahora es el Central Vicente Cáceres que estaba en el centro de Tegucigalpa, ahí por el Congreso Nacional, el director era el profesor Saúl Zelaya Jiménez. Entrevista a Entrevista a Darguin Amaya, código de entrevistado: SAAM3, Tegucigalpa M.D.C, 19 de octubre 2014. En su mayoría, los adultos mayores no tienen ninguna jubilación, ni pensión, debido a que no tuvieron un trabajo que les brindara este beneficio y aunque varios adultos han trabajado durante muchos años, se han retirado sin ningún beneficio o compensación de retiro. Dentro de los que se retiran, hay quienes lo hicieron voluntariamente a causa de limitaciones derivadas de su salud o porque desean iniciar su descanso.

También dentro de los adultos mayores hay quienes necesitan seguir en el mercado laboral para costear su subsistencia, pero que desisten de buscar trabajo ante la discriminación sufrida por la edad y algunas desventajas que propicia el mercado con respecto a personas más jóvenes y con más calificación en un mercado con altos niveles de desocupación (No, yo trabajé muchos años, pero no me dieron nada de eso, ni jubilación, ni pensión; entrevista a Entrevista a Darguin Amaya, código de entrevistado: SAAM2, Tegucigalpa M.D.C, 19 de octubre 2014.

La mayoría de los adultos que residen en los hogares de ancianos no perciben ningún ingreso y en algunos acasos están internos y sin pagar nada en el centro; otros, aunque tengan familia, esta se olvida por completo de ellos. Es en estas circunstancias en donde se ve la necesidad de la familia; al respecto, este estudio evidencia que los adultos mayores internos en los hogares de ancianos en Tegucigalpa tienen sentimientos de soledad por encontrarse separados de su núcleo familiar, mostrándose apáticos, poco participativos en las actividades programadas, con mayores factores de riesgo como sedentarismo y aislamiento (No, no tengo familia, solo tuve un hijo al que le di todo desde niño, ahora es periodista y no se acuerda de mí, ni pregunta por mí, yo lo miro porque sale en televisión y viera que me alegra que esté bien y que esté luchando, pero quisiera verlo; entrevista a Entrevista a Darguin Amaya, código de 
entrevistado: MEAF3, Tegucigalpa M.D.C, 24 de noviembre 2014.

Un dato importante en este estudio es que entre los pocos familiares que visitan y atienden a los adultos mayores en los hogares de ancianos son las hermanas y las hijas, quienes se encargan del cuidado y de cubrir las necesidades de estos. Tales antecedentes evidencian la relevancia del rol de la familia en el cuidado de un adulto mayor, pues son fundamentalmente para cubrir necesidades afectivas. Así, la responsabilidad de los hijos sobre el cuidado de los padres no solo debe ser un aspecto moral, sino que también es un deber (Sí, mis hermanas vienen, así los fines de semana, aunque sea un ratito; Entrevista a Darguin Amaya, código de entrevistado: SAAF1, Tegucigalpa M.D.C, 19 de octubre 2014.

Se ha logrado identificar que el personal que atiende a los adultos mayores es personal capacitado y con experiencia (Yo soy enfermera y atiendo a los adultos y tengo 9 años de trabajar aquí; Entrevista a Darguin Amaya, código de entrevistado: HPEM1, Tegucigalpa M.D.C, 07 de diciembre 2014. Se desenvuelven cubriendo las necesidades de los adultos, los acompañan a los cuando realizan visitas al médico u hospitales, gestionan fondos para beneficio de los adultos mayores en los hogares de ancianos, trabajan muchas horas del día y en fines de semana; asimismo, a medida que van conociendo a los adultos les van tomando mayor cariño y afecto (Yo trabajo en aseo, bañando a los adultos que no pueden asearse solos, cuidándolos, dándoles comida a los enfermos, dándoles medicamentos y en lo que ellos necesiten; Entrevista a Darguin Amaya, código de entrevistado: SAEM1, Tegucigalpa M.D.C, 07 de febrero 2015 .

Se ha identificado que en su mayoría el personal que cuida del adulto mayor dentro de los hogares de ancianos son mujeres y solamente hay varones en el área de vigilancia en los portones y conserjes, por lo que el personal de enfermería dice que cuando una persona está enferma, en cama, necesitará ayuda para muchas actividades cotidianas que todos consideramos normales, tales como lavarse, cepillarse los dientes e ir al baño. Algunas personas incapacitadas solo necesitan asistencia mínima, quizá darles la mano para ayudarles a entrar a la regadera, mientras que otras necesitan de otra persona que se encargue de atender todas sus necesidades físicas cotidianas. La persona enferma también necesita que la pongan cómoda, comprobar que tenga suficientes almohadas y que no tenga mucho calor, ni pase frío.

Con respecto a la pregunta de adónde acude cuando necesita atención médica y quién le acompaña, en su mayoría los adultos mayores son atendidos en el mismo hogar de ancianos, ya que llega algún médico general o se auxilian de la enfermera 
que les cuida, son pocos los adultos que tienen seguro social y en algunos casos expresan que han estado internos y se han quedado solos en el centro hospitalario, solamente los llegan a traer en ambulancia (No, aquí en el centro hay una enfermera y nos da medicamentos, cuando necesitamos ir al doctor, vamos a un particular o al hospital escuela, porque no tengo seguro; entrevista a Entrevista a Darguin Amaya, código de entrevistado: HPAF2, Tegucigalpa M.D.C, 07 de diciembre 2014.

El estado de salud física y mental de las personas mayores depende en gran parte de la forma de alimentarse en la infancia y la edad adulta. En la calidad de vida de los adultos mayores influyen los hábitos de alimentación y otros factores de tipo afectivo que determinan la seguridad alimentaria y nutricional de este grupo de población, tales como la soledad, la falta de recursos económicos, la baja disponibilidad de alimentos y las enfermedades crónicas, entre otras expresadas por los adultos mayores.

Además de los cambios físicos, la persona que envejece se enfrenta a cambios. En lo referente a los hábitos alimentarios de los adultos mayores se encontró que la mayoría consume tres comidas al día o más, sin embargo, en un hogar de ancianos solo se consumen dos comidas al día, lo que implica un riesgo de déficit en el consumo de calorías y nutrientes que contribuye a una adecuada dieta alimentaria. Al indagar por las comidas que hacen los adultos mayores, se encontró que las de mayor consumo son el desayuno, el almuerzo y la cena; en algunos casos tienen merienda cuando llegan a visitarlos. Llama la atención que algunos adultos no consumen la última comida del día debido a algunos problemas de salud (Aquí comemos varias cosas, el pollo y arroz es lo que más comemos; Entrevista a Darguin Amaya, código de entrevistado: MEAF2, Tegucigalpa M.D.C, 24 de noviembre 2014.

Como resultados del componente cualitativo se destaca que los adultos mayores reconocen que a su edad se limita el acceso a los alimentos por diferentes factores, aspecto que deteriora su estado de salud y nutrición. Muchos de los adultos mayores al no tener ingresos, dependen de otras personas que gestionan los alimentos.

Los adultos mayores en su mayoría expresan que hacen algún tipo de actividad solamente cuando tienen visitas de centros educativos que los ponen a hacer ejercicios, bailes y celebraciones de la palabra de Dios (Lo que hago para pasar ocupada es costura, bordado, el rosario y así en la mañana nos ponemos a rezar en grupo, también vienen grupos y nos ponen a hacer actividades; Entrevista a Darguin Amaya, código de entrevistado: SAAF1, Tegucigalpa M.D.C, 19 de octubre 2014. 
Se les preguntó a los adultos mayores sobre sus sentimientos de soledad, desesperanza, ansiedad o depresión; en su mayoría expresan que tienen estos sentimientos en varias ocasiones como al ver que llegan familiares a visitar a otro adulto en el centro o cuando llega alguna visita al centro y luego se regresa; también cuando necesitan ayuda económica o algún medicamento y no lo pueden conseguir (Por las tardes y en las noches también me acuerdo de cosas y de la familia, pero me pongo a rezar por todos y ahí es cuando me cuesta dormir; Entrevista a Darguin Amaya, código de entrevistado: HPAF3, Tegucigalpa M.D.C, 07 de diciembre 2014.

En esta investigación se observó que la mayoría de los adultos muestran una salud emocional positiva, aunque esto es relativo, debido a que en comparación con su estilo de vida antes de llegar al hogar de ancianos, estar internos es buena calidad de vida, según lo expresado por algunos de los entrevistados que vivían solos, en la calle, sin alimentación, sin abrigo y faltos de afecto. Es interesante observar algunos aspectos que pueden ser considerados factores desencadenantes de la soledad, tales como abandono de muchos de sus intereses o actividades de la vida diaria y sentir que su vida está vacía (Tengo una buena calidad de vida, no me puedo quejar con lo que Dios me da; Entrevista a Darguin Amaya, código de entrevistado: MEAM1, Tegucigalpa M.D.C, 24 de noviembre 2014

Existen diferentes razones por las cuales los adultos mayores están ingresados en los hogares de ancianos; en su mayoría dicen que es porque no tienen quien les cuide, ni familia, aunque es la misma familia la que muchas veces los ingresa al hogar (Un sobrino me trajo porque me di una caída y no había quien me cuidara en casa; Entrevista a Darguin Amaya, código de entrevistado: HPAF1, Tegucigalpa M.D.C, 07 de diciembre 2014.

En otros casos expresan que debido a su enfermedad no tienen quien les cuide, también si no tienen trabajo, ni casa donde vivir o alguna organización que los ampare, los llevan al hogar de ancianos (Me trajeron los hermanos de la comunidad, yo pertenezco al neocatecumenado en la Medalla Milagrosa y no tenía ningún familiar que me cuidara; Entrevista a Darguin Amaya, código de entrevistado: MEAF3, Tegucigalpa M.D.C, 24 de noviembre 2014. Además, otra causa encontrada es porque vivían en la calle o no tenían quien le brindara casa y comida (Me trajeron del Chiverito, ahí vivía en la calle y sin comida; Entrevista a Darguin Amaya, código de entrevistado: CEAM1, Tegucigalpa M.D.C, 19 de diciembre 2014.

Es relevante el rol que ejerce un cuidador del adulto mayor, pero más importante es si este pertenece a su núcleo familiar. Los adultos expresan una gran necesidad de la 
familia para ser escuchados y tener la confianza de expresarse y pedir el apoyo que necesitan.

\section{CONCLUSIONES}

1. Los adultos mayores que residen en los hogares de ancianos, en su mayoría, consideran que tienen una buena calidad de vida; en algunos casos relacionan esta calidad de vida con la salud, la alimentación o trato recibido, lo comparan con la calidad de vida que vivían antes de llegar a los hogares de ancianos.

2. Los adultos mayores que residen en los hogares de ancianos en su mayoría no tienen familia y los que tienen algún familiar poco los visitan; en algunos casos reciben apoyo económico y moral, para los demás adultos las necesidades son cubiertas por el hogar de ancianos. Estos adultos van quedando solos, sus amigos y familiares los abandonan en algunos casos y en otros porque debido a su edad ya no tienen familiares cercanos, ni amigos.

3. El perfil del recurso humano que atiende y cuida de los adultos mayores en los hogares de ancianos en su mayoría tiene mucha experiencia cuidando adultos, trabajan desde hace muchos años en los hogares y les toman amor y cariño a los adultos mayores y a su trabajo con ellos.

4. Los adultos mayores en los hogares de ancianos tienen diferentes necesidades. Se identificó la falta de productos básicos como: jabón de baño, papel higiénico, ropa interior, vestuario, zapatos, pañales, meriendas, medicamentos, falta de atención médica, espacios para recreación y espacios físicos para alojar más adultos.

5. Los adultos mayores expresaron que hay diversas causas por las que han llegado a los hogares de ancianos, entre ellas está el no tener familia, ni quien los cuide en casa; no tienen trabajo, ni casa, porque están enfermos. Vivían en la calle para tener compañía y donde comer. Existe una concordancia entre lo expresando por los adultos mayores, lo que se observó y expresaron los empleados y directores de los hogares de ancianos en cuanto a la calidad de vida de los adultos mayores; igualmente sobre lo que les está afectando para tener una buena calidad de vida. 


\section{RECOMENDACIONES}

1. Ante el rápido crecimiento de la población adulta mayor que se refleja en los últimos años, se debe brindar una mayor importancia a la prevención del abandono de las personas mayores y promover un proceso de envejecimiento más saludable.

2. Es esencial que el adulto mayor participe en las actividades de la familia y la sociedad, es necesario generar políticas para que la población adulta mejore su calidad de vida a través de programas que estimulen la participación activa de la persona mayor en la vida comunitaria, mediante cambios en los estilos de vida.

3. A los empleados y directores de hogares de ancianos, por ser quienes mantienen un contacto directo con esta población, que contribuyan a coordinar actividades que realicen los adultos mayores con el objetivo de ocupar su tiempo libre, realizar trabajo creativo y participativo que mejore su salud integral.

4. A la sociedad, en general, porque en el mejor de los casos todos seremos algún día adultos mayores, para que brinden todo el apoyo necesario para cubrir sus necesidades físicas, sociales, económicas y emocionales a sus familiares que se encuentran en la etapa de la vejez.

5. Que las autoridades gubernamentales y la sociedad hondureña pueda crear condiciones económicas, sociales, políticas y culturales para que todas las personas dentro de la familia puedan fortalecer su autoestima y autonomía, con una política que permita fortalecer los factores protectores, desde la niñez, para alcanzar en la vejez una vida digna.

\section{AGRADECIMIENTOS}

Al doctor Elvis Trejo, asesor técnico, por haberme brindado la oportunidad de poner en práctica muchos conocimientos. A la Universidad Nacional Autónoma de Honduras, a través de la Dirección de Investigación Científica y Posgrado, a la Maestría en Metodologías de Investigación Económica y Social por impulsar la educación en investigación en Honduras. 


\section{BIBLIOGRAFÍA}

Balestrini, M. (2002). Cómo se elabora el proyecto de investigación. Caracas: BL Consultores Asociados.

Yuni, José y Urbano, Claudio. (2003). Recursos Metodológicos para la Preparación de Proyectos de Investigación. Córdoba -Argentina: Editorial Brujas.

Castro, R. (2011). Teoría social y salud. Buenos Aires: El Lugar Editorial y CRIM.

Báez, Juan y De Tudela, Pérez. (2009). Investigación Cualitativa, 2da Edición. Madrid: ESIC Editorial.

Escribano, A. (2007). El proceso de investigación social cualitativo. Buenos Aires: Prometeo Libros.

Mejía Navarrete, J. (2002). Infancia y ciencia social. Lima, Perú: Editorial Universidad Nacional Mayor de San Marcos.

Yuni, José y Urbano, Claudio. (2006). Técnicas para investigar 2. Córdoba -Argentina: Editorial Brujas 